This is an Open Access article distributed under the terms of the Creative Commons Attribution-Noncommercial License, which permits unrestricted use, distribution, and reproduction in any noncommercial medium, provided the original work is properly cited.

\title{
SPICA AND THE UPCOMING REVOLUTION IN RADIO ASTRONOMY
}

\author{
Huub Röttgering \\ Leiden Observatory, Leiden University, P.O. Box 9513, 2300 RA Leiden, The Netherlands
}

\begin{abstract}
The new radio telescopes that are currently coming on line such as LOFAR and e-VLA and - in the somewhat more distant future - SKA, will revolutionize radio astronomy. Among many other things, they will enable observations of unprecedented detail of events where energetic particles and magnetic fields play a dominant role. This is the case for, for example, radio jets, supernovae and large scale shocks in galaxy clusters. SPICA will enable observations of crucial stages of the formation of both stars and galaxies. During the last years it has become clear that the interplay between highly energetic events and the more quiescent phenomenon related to the accretion of cold gas plays a crucial role in the formation and evolution of galaxies and their associated AGN, and clusters of galaxies. In this contribution we will review why the combined observations of future radio observatories and SPICA will advance our understanding in this field.
\end{abstract}

Key words: Galaxies: formation - Stars: formation - Missions: SPICA

\section{INTRODUCTION}

During the last decades it has become clear that in order to advance our understanding of the formation and evolution of stars, galaxies and AGN, multi-wavelength observational studies are essential. An obvious reason is that there are many types of gas, dust, stars and AGN in the Universe that often have key diagnostics visible at distinctly different wavelength regimes. A second reason is that an understanding of certain astrophysical phenomena is often only acquired when the interaction between very different physical mechanisms is taken into account. A very nice example is the interplay between information obtained with far-infrared and radio telesopes that each probe very different processes. Far-infrared telescopes primarily observe continuum and line emission from gas and dust associated with the formation of stars, where as radio telescopes chart relativistic synchrotron emission associated with energetic events such as jets emerging from massive black holes at the centre of galaxies. In this case the interplay might be very intimate: on the one hand gas and dust ignite the activity of the blackholes, while the resulting AGN energy output might regulate the rate of starformation in the hosting galaxy. Only semi-analytic models that take this "feed-back" into account seem to be able to reproduce a number of global characteristics of the entire galaxy population such as the galaxy luminosity function and its evolution (Croton et al., 2006).

In this contribution, we will first briefly discuss the SKA radio telescope and how it will impact on many important areas of current astrophysics in about 10 years from now. In the years before SKA will come on-line, a number of new important facilities will collectively pave the way for SKA, both in a technical and scientific sense. One of these instrument that we will briefly touch upon is LOFAR, a revolutionary radio telecope that will observe from $240 \mathrm{MHz}$ down to the lowest accessible frequencies from the ground. In the second part of this contribution we will discuss how projects combining SPICA and radio data will be particularly valuable for studies of AGN, clusters and distant galaxies.

\section{SKA RADIO TELESCOPE}

The Square Kilometre Array will play a major role in answering key questions in modern astrophysics and cosmology (Schilizzi et al., 2008; Lazio, 2009). Its design is driven by five key science areas that, combined, dictate the frequency range to cover from $70 \mathrm{MHz}$ up to $>25 \mathrm{GHz}$ and a collecting area of one million square metres. Further requirements include enormous survey speed and very high dynamic range. A radio telescope with all these capabilities will be a very versatile instrument that is not only capable of carrying out the very ambitious driving science goals, but that will also address issues related to virtually the entirety of astronomy. Although no detailed design has been finalized, it is likely that SKA will have a centrally condensed scale-free array configuration, with $50 \%$ of the collecting area in the inner $2.5 \mathrm{~km}$ and baselines of up to $3000 \mathrm{~km}$. Currently it is foreseen that SKA will be built in 3 phases, with each phase providing an instrument with significant scientific capabilities. At the end of phase 3, SKA will attain its full capability. This is currently foreseen to be achieved in the 2020 timeframe. The 5 key science areas are:

- Probing the Dark Ages 
The aim is to probe structures in the universe at an epoch when the Universe made the transition from largely neutral to its largely ionized state today. Very deep observations should be capable of imaging the 21 $\mathrm{cm}$ line emission and absorption of individual regions that make this transition. At shorter wavelengths, SKA should be capable of detecting species such as CO, $\mathrm{HCN}$ and $\mathrm{HCO}^{+}$in distant starbursting galaxies to constrain both the dynamical state and the physics of the young galaxies.

- Galaxy Evolution, Cosmology and Dark Energy

The aim here is to detect a significant fraction of all the galaxies to a redshift of $z \sim 2$, with the aim of mapping out the $3-\mathrm{D}$ cosmic web from the present day up to the epoch when most of the galaxies were forming. This will give an unbiased view of the evolution of star formation rates in galaxies, free from dust obscuration. Furthermore, it will provide a very clean measurement of "acoustic oscillations", yielding an extremely accurate determination of how the equation of state of dark energy evolves with cosmic time.

- The Origin and Evolution of Cosmic Magnetism

Deep radio observations will be used to constrain the magnetic field properties in many classes of object such as galaxies, clusters of galaxies, and AGN. This is essential to elucidating the role that magnetic fields play in shaping the evolution these objects.

- Strong Field Tests of Gravity Using Pulsars and Black Holes

With very high-precision timing measurements of pulsars, the laws of the nature of space and time as formulated by Einstein can be tested to their limits.

- The Cradle of Life

The aim here is (i) to image planet-forming disks at high angular resolution, (ii) to study prebiotic molecules, and (iii) to search for technological civilisations on habitable planets.

\section{SKA-PATHFINDERS}

The technical and organisational challenges that face SKA are significant and a phased approach is clearly needed to address these challenges. To advance and test out the required technologies and to carry out important "SKAtype" science, a number of radio telescope projects have started. These "Path-finder projects" can be dividied into three groups. First, there are projects that aim to observe a large fraction of the sky in the $\mathrm{GHz}$ regime making use of a fairly large number of relavely small dishes. These projects include the South-African Meerkat project (50 or more dishes of $12 \mathrm{~m}$ diameter), the Australian ASKAP project ( 26 dishes of $12 \mathrm{~m}$ ) and the USA-based Allen Telescope Array (350 dishes of $6.1 \mathrm{~m}$ ). Second, there are a number of projects that aim to detect the epoch of reionisation through the $21 \mathrm{~cm}$ line. There include: MWA (Australia), Core (Australia), Paper (USA), 21CMA (China). Third, the LWA is dedicated to observe the Universe with high angular resolution at frequencies below $<100 \mathrm{MHz}$. The radio telescope LOFAR aims to carry out important work in the areas two and three and is described in more detail in the following section.

\section{LOFAR}

LOFAR, the Low Frequency Array, is a next-generation low frequency radio telescope that is currently under construction. Its revolutionary design makes use of phased array technology that gives the critical advantage of delivering an affordable telescope with the necessary large effective aperture. LOFAR will have low frequency antennas optimized for the $30-80 \mathrm{MHz}$ range and high frequency antennas which have their maximum sensitivity between 115 and $240 \mathrm{MHz}$. These antennas are being grouped together in stations that are the size of a typical soccer field. The electric signals from the antennas will be digitized and appropriate delays applied so that station beams on the sky can be formed. Currently 18 stations are planned in a central region of $2 \times 3 \mathrm{~km}$, with another 18 to form baselines of up to $100 \mathrm{~km}$, and at least 6 stations in countries in Europe (Germany, UK, Sweden, and France). The current time-line is to have all these station on-line in 2010. The design of LOFAR has been driven by 4 key scientific topics:

\section{- Epoch of reionisation.}

The main aim is to determine the angular and spectral power spectrum of the reionisation signal.

- The formation and evolution of clusters, galaxies and black holes.

LOFAR surveys at the key frequencies 15, 30, 60, 120 and $200 \mathrm{MHz}$ will provide a goldmine for investigating several fundamental questions relating to the formation of massive black holes, galaxies and clusters of galaxies.

\section{- Transient Sources}

LOFAR's large instantaneous beam allows for unique surveys of radio transients. The available resolution will be sufficient for the crucial task of rapid optical and X-ray identifications. Key targets will be gammaray bursts, exo-planets and pulsars.

\section{- Ultra High Energy Cosmic Rays}

Cosmic ray particles produce extensive airshowers in the atmosphere. LOFAR will constrain their energies, composition and origin on the sky. 
In addition to these 4 driving science cases, both the Sun and cosmic magnetism has been recognised as topics for which LOFAR will carry out important observations. Finally, with LOFAR's capability to undertake observations at a frequency window on the Universe that has not yet been explored with modern telecopes, its parameter space for serendipitous discovery is relatively large (e.g. Fabian, 2009). This is particularly the case for the very low frequency radio observations and the all-sky monitoring capability that LOFAR provides.

\section{LOFAR SURVEYS}

The proposed LOFAR surveys are designed to advance our understanding of the formation and evolution of galaxies, AGNs and galaxy clusters and to leave a lasting legacy to the global astronomical community. Because of its low operating frequencies, and the resulting large field of view on the sky, LOFAR is an ideal survey facility. For example, at $30 \mathrm{MHz}$ each beam has a $10 \mathrm{deg}$ field of view. With theoretical LOFAR sensitivities and feasible observing times, such a field will typically contain $1 z>6$ radio galaxy, 10 Abell clusters, 10 NGC galaxies, 10 lensed radio sources and several giant $(>1 \mathrm{Mpc}$ ) radio galaxies. The legacy value of such LOFAR surveys will be comparable to previous high-impact surveys (e.g. Palomar, IRAS, SLOAN, GALEX, Spitzer, NVSS). Our LOFAR surveys will complement currently planned surveys in other wavebands (e.g. JEDAM, Euclid, Pan-STARRS, Herschel, Planck, VISTA, VST, E-VLA, ASKAP, MEERKAT, ATA). These surveys will provide metre-wave data on up to $10^{8}$ galaxies and $10^{4}$ clusters out to $z \sim 8$, and will address a wide range of topics from current astrophysics.

The area and depth of the LOFAR surveys have been designed to optimise the scientific yield for three topics:

\section{- High-redshift radio galaxies}

High-redshift radio galaxies (HzRGs, $z>2$ ) are unique laboratories for studying the formation and evolution of massive galaxies, rich clusters and massive black holes. (see review by Miley and De Breuck, 2008). Focusing on rare ultra-steep spectra (USS) radio sources has resulted in the discovery of most known $z>2$ HzRGs (Röttgering et al., 1997; de Breuck et al., 2002). Presently, the most distant HzRG has $z=5.1$. Because of its low operating frequency, LOFAR will detect large numbers of USS HzRGs. This should push studies of massive galaxies and protoclusters to $z \sim 8$. Our goal is to detect 100 radio galaxies at $z>6$ to enable robust studies of the properties of $z>6$ massive galaxies and protoclusters and provide sufficient number of radio sources within the Epoch of Reionization to facilitate $\mathrm{HI}$ absorption studies.

\section{- Galaxy clusters}

Clusters often contain Mpc-sized diffuse radio sources that are not clearly associated with individual galaxies. Diffuse radio cluster sources are classified into either amorphous "halos" located close to the centres of clusters, or elongated "relics" located near the cluster peripheries. (e.g. Giovannini \& Feretti, 2004). Currently, such diffuse radio sources are known in about 30 nearby rich galaxy clusters and these often have ultra-steep spectra. With its unprecedented low-frequency sensitivity, LOFAR should detect many thousands of diffuse cluster radio sources out to $z \sim 0.8$. Our survey goal is to detect about 100 clusters at $z \gtrsim 0.6$ and to study 60 local clusters in unprecedented detail. This sample will enable a robust study of magnetic fields, plasma shocks and particle acceleration mechanisms as a function of cluster properties and redshift. Also detailed studies of the radio galaxies, quasars and tailed sources in these clusters will provide important information on the overall thermal balance and star formation in clusters.

\section{- Cosmic star formation history}

The deepest surveys will detect radio emission from millions of regular star-forming galaxies at the epoch when the bulk of galaxy formation occured. Galaxies with extreme star formation rates will be detectable out to the epoch of reionisation. The combination of LOFAR and infra-red surveys will yield radio-IR photometric redshifts, enabling studies of the volume-averaged star formation rate as a function of epoch, galaxy type and environment. Such studies need surveys that cover a sky area large enough to sample diverse environments (from voids to rich proto-clusters) over a wide range of cosmic epochs. Our surveys are planned to contain 50 proto-clusters at $z>2$, sampling the most extreme (and rarest) peaks in the density field.

\section{SPICA AND FUTURE RADIO PROJECTS}

Very simplistically, SPICA will measure properties of relatively cold gas and dust in distant galaxies and AGN. A significant fraction of this gas is cooling down to form stars and feed AGN. Again, very simply, AGN heat up the gas in galaxies and clusters. This heating periodically suppresses the rate of star formation in massive galaxies, supposedly leading to local ellipticals that are "red and dead". In clusters it is the radio-loud AGN in particular that influence the total energy balance in the gas, and heat up at least part of the gas to reduce or even stop cooling flows. It is the interplay and balance between these cooling and heating mechanisms that plays an important role in shaping the size, mass and composition of galaxies and clusters of galaxies (e.g. Figure 1).

While SKA will have the capability of observing virtually every galaxy that SPICA will detect, and also measure its redshift, the SKA is likely to start operating well after SPICA has flown. LOFAR will have carried out a significant fraction of its surveys before the launch of SPICA. In Figure 2, we indicate the flux density at $150 \mathrm{MHz}$ as a 


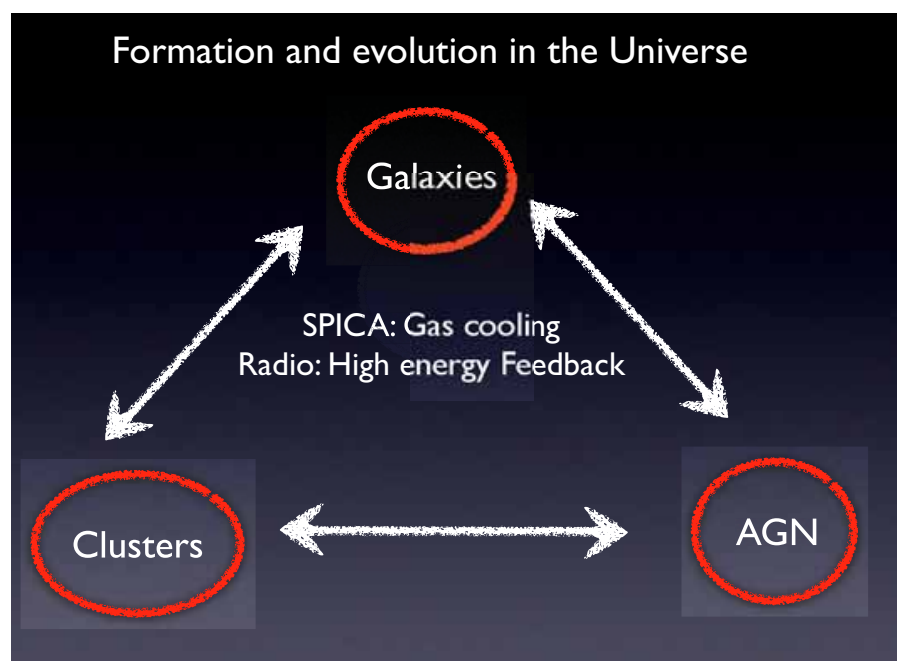

Figure 1. A schematic view of how heating and cooling mechanisms are important in shaping the properties of important constituents in the Universe.

function of redshift for galaxies with star formation rates as indicated. Also marked are the expected depth of LOFAR surveys that cover the entire sky (Tier-1), about a thousand square degrees (Tier-2) and only a selected number of pointings, but extremely deep (Tier-3). Finally, indicated are representive depths of deep surveys with SPICA and a number of upcoming radio telescopes. A conclusion is that LOFAR and SPICA can both detect galaxies with a star formation rate of $20 \mathrm{M}_{\odot} /$ yr up to $z \sim 2$ and $500 \mathrm{M}_{\odot} /$ yr up to $z \sim 6$.

With this in mind, we now consider a list of projects that address issues related to the formation and evolution of galaxies and clusters and the role that the cooling and heating mechanisms play.

\subsection{The ALIGNMENT EFFECT IN $z \sim 1$ RADIO GALAXIES}

One of the reasons that powerful radio galaxies at high redshift (High- $z$ Radio Galaxies, HzRGs) are fascinating objects to study is the interesting interplay between many of their different components. To illustrate this, we show in the upper panel of Figure 3 an HST image of one of the archetypical powerful radio galaxies at $z \sim 1,3 \mathrm{C} 266$, overlaied by a 0.2 arcsec resolution VLA image taken at 8.4 $\mathrm{GHz}$ (from Best et al. 1996). Furthermore, we show in the lower panel of Figure 3 a cartoon of some of the important components of a radio galaxy. The fuzzy knotty structure visible in the HST image located between the two radio lobes is clearly aligned in the direction of the radio lobes. Many suggestions have been made as to the origin of this commonly called "alignment effect". The suggestions that have been put forward include young stars formed when the radio jet passed through the ISM, scattered light from particles (dust or electrons) located within a cone of light emitted by a hidden quasar and nebular continuum emit-

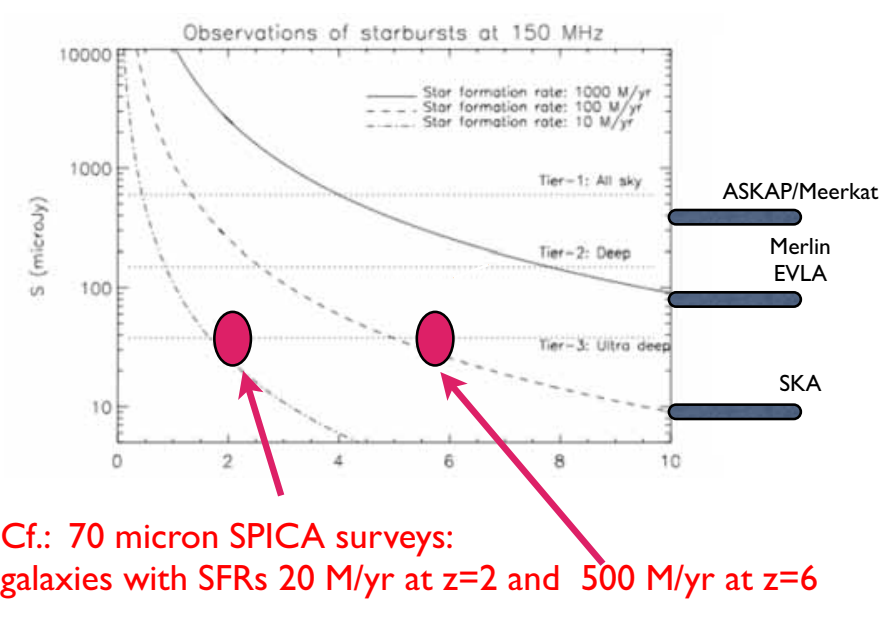

Figure 2. Flux density at $150 \mathrm{MHz}$ as a function of redshift for galaxies with a starformation rate as indicated. Also shown are the expected depths of LOFAR surveys that cover the entire sky (Tier-1), about a thousand square degrees (Tier-2) and only a selected number of pointings, but extremely deep (Tier-3). Finally, indicated are representive depths of deep surveys with SPICA and a number of upcoming radio telescopes.

ted by the ionised gas which is surrounding such objects (see McCarthy 1993) The aligned light is mainly seen for smaller radio galaxies, rather than for large radio galaxies. This indicates that the properties of the stars and the ISM strongly change when the jets makes its way out of the galaxies. SPICA will measure in detail how the physical properties of the dust and gas change along the main axis of the optical light, allowing us to study whether the properties of the ISM changes on the times-scales that the jets leave the galaxy $\left(10^{6-7}\right.$ years $)$.

\subsection{ENERGY BALANCE IN CLUSTERS}

During cluster mergers an enormous amount of energy is released. This energy can heat the intra-cluster medium and accelerate particles to highly relativistic energies. In the presence of magnetic fields these particles will emit diffuse synchrotron radiation, known as radio relics and halos (see the reviews by Ferrari et al., 2008, and references therein). An interesting case is the complex merging cluster MACS J0717.5+3745. Recent GMRT observations at $610 \mathrm{MHz}$ showed diffuse steep spectrum radio emission at a location roughly coinciding with regions in the cluster having higher temperatures (Figure 4). This shows that the radio emission is probably the result of a largescale shock wave within the cluster, where particles are accelerated. With its excellent sensitivity to diffuse steep spectrum radio emission, LOFAR will detect many thousand of such systems (e.g. Cassano). SPICA spectrosopy will trace major cooling lines like [OI], [OIII], [NII], [CII] and [SiI]. The combination of LOFAR and SPICA will map out the regions of heating and cooling of the X-ray 

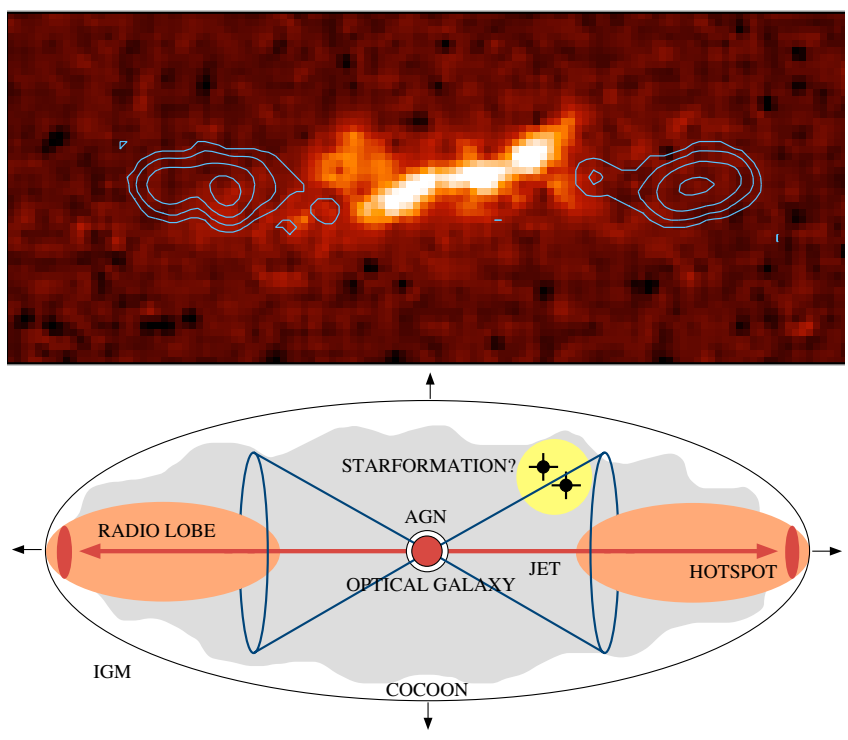

Figure 3. (Top) The 0.2 arcsec-resolution $8.4 \mathrm{GHz}$ VLA radio image (contours) and the ( R-band) HST image (greyscale) of the $39 \mathrm{kpc} z=1.272$ powerful radio galaxy 3C266 (from Best et al. 1976). The image has been rotated so that north is towards the left. (Bottom) A cartoon indicating some of the components of distant radio galaxies.

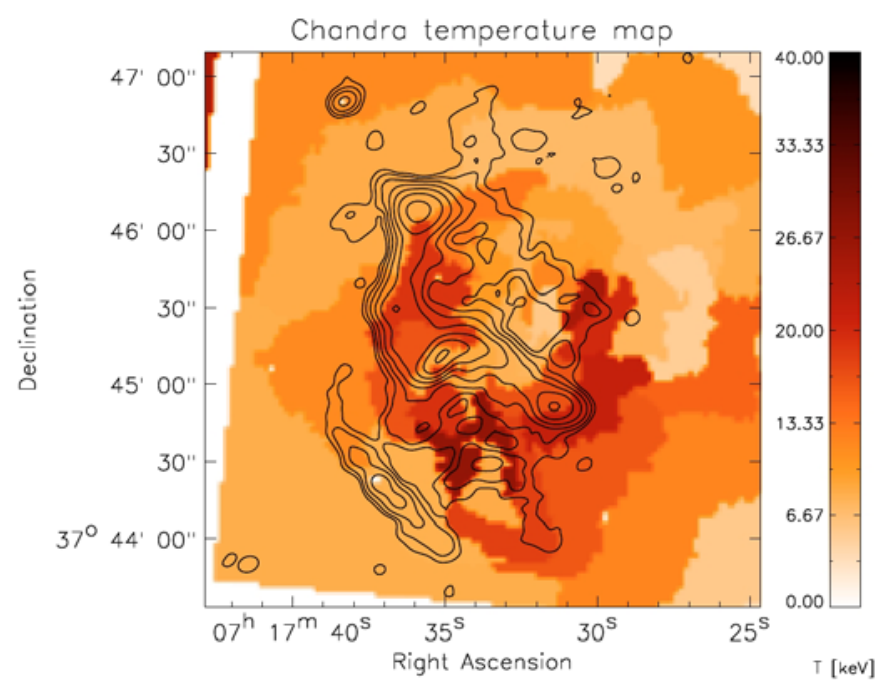

Figure 4. Temperature map ( overlaid with $610 \mathrm{MHz}$ GMRT radio contours. Contour levels are drawn at $[1,2,4,8,16,32, \ldots] \times$ $0.312 \mathrm{mJy} /$ beam 1. The beam size is $8.2^{\prime \prime} \times 6.0^{\prime \prime}$ (figure taken from Ma et al., 2009 and van Weeren et al., 2009)

gas, crucial for our understanding energy balance in the $\mathrm{X}$-ray gas.

\subsection{AcCretion Processes in AGN}

As already discussed, there is increasing evidence that many physical relations between AGN, galaxies and the large scale structures have to be taken into account if their formation and evolution is to be understood. Recently, we have studied aspects of these relations on the basis of XMM-Newton, CFHTLS, Spitzer and radio surveys in the XMM-LSS field. The radio surveys we have carried out with the Very Large Array are at 74 and $325 \mathrm{MHz}$, and with the Giant Meterwave Radio Telescope (GMRT) at 230 and $610 \mathrm{MHz}$ (Tasse et al., 2008).

The results clearly show that there are two groups of radio galaxies. The first group resides in massive galaxies, does not show signs of infrared excess due to a torus and are preferentially found in cluster environments. The second group consists of radio galaxies with less massive hosts, that show infrared torus emission, and that are located in large scale underdensities. Finally, X-ray selected AGN show infrared torus emission and are preferentially found in environments underdense on large scales.

These results are interpreted as AGN being fed by two different types of accretion: the we have studied aspects of these relations on the basis of XMM-Newton, CFHTLS, Spitzer and radio surveys in the XMM-LSS field. The radio surveys we have carried out with the Very Large Array are at 74 and $325 \mathrm{MHz}$, and with the Giant Meterwave Radio Telescope (GMRT) at 230 and $610 \mathrm{MHz}$ (Tasse et al., 2008).

The results clearly show that there are two groups of radio galaxies. The first group resides in massive galaxies, does not show signs of infrared excess due to a torus and are preferentially found in cluster environments. The second group consists of radio galaxies with less massive hosts, that show infrared torus emission, and that are located in large scale underdensities. Finally, X-ray selected AGN show infrared torus emission and are preferentially found in environments underdense on large scales.

These results are interpreted as AGN being fed by two different types of accretion: the Radio- or Hot mode versus the Quasar- or "Cold mode". In this picture, the quasar mode is radiatively efficient, and is caused by accretion of cold gas onto the super-massive black hole, while the radio mode is radiatively inefficient and results from the accretion of hot gas.

The combination of deep radio and SPICA surveys will determine how these relations change up to redshifts of at least two. Important questions include: does the fraction of radio-to-quasar mode accretion change with redshift? Does the environment at high redshift as dominant an effect as it does locally?

\subsection{Proto-Clusters}

Galaxy clusters are unique, high-density regions in the Universe and are therefore important test beds for theories of galaxy formation and evolution. Although early theoretical work indicated otherwise (Bahcall \& Fan 1998), the last decade of observational studies have shown surprising little evolution in the physical properties of clusters between $z \sim 1$ and the present epoch. The observational 
challenge therefore was to find and study clusters that could be regarded as proto-clusters at much higher redshifts than previously was deemed necessary. Both their extreme faintness and sparseness on the sky necessitated the development of new observing techniques. In Leiden we pioneered the search for proto-clusters associated with high redshift radio galaxies (HzRGs). With radio galaxies being among the most luminous, most massive galaxies known at high redshifts at almost any wavelength, they proved to be successful hunting grounds for the search for proto-clusters. Almost 10 proto-clusters have now been identified.

The next crucial step in this area of research is to define and study large, unbiased samples of proto-clusters. Current high frequency radio and X-ray survey techniques lack the sensitivity to probe the required faint levels whilst observations at wavelengths such as optical, near-IR and sub-mm have difficulty covering the large area on the sky needed to obtain a sample of proto-clusters. LOFAR, in combination with SPICA, will allow a good sample of proto-clusters up to $z \sim 3$ to be defined. The various galaxy populations in these clusters will be studied and their masses, ages, sizes, star formation rates, and location in the cluster will be characterised. The observations of the galaxy and cluster properties will be directly compared with the semi-analytical galaxy formation models. This is likely to lead to significant improvements of the modelling of star formation, galactic winds, chemodynamics and radiative cooling.

\subsection{RADIO GALAXIES AT THE EPOCH OF REIONISATION}

With radio luminosities of up to a thousand times greater than our own galaxy, luminous distant radio galaxies are unique cosmic probes. Even very distant radio galaxies are thought to host extremely massive black holes $\left(10^{9}\right.$ solar masses) and are therefore important laboratories for probing phenomena in the early Universe ultimately related the accretion of matter onto the first rotating massive black holes. Secondly, they are among the brightest, most massive, and gas rich galaxies known in the early Universe (De Breuck et al., 2002; Seymour et al., 2007) and can therefore be studied in far greater detail than "normal" galaxies at these enormous distances Thirdly, high redshift radio galaxies are excellent targets in a search for the ancestors of massive clusters, proto-clusters.

Since extremely distant radio galaxies are relatively bright at very low radio frequencies, LOFAR's capabilities are ideal to efficiently pick out radio galaxies at larger distances than possible with present radio telescopes. LOFAR should be able to discover a few hundred radio galaxies at distances significantly larger than the most distant radio source currently known (TN J0924-2201 at $\mathrm{z}=5.2$, van Breugel et al. 1999). Particularly important is that these sources will be located close to, or even at, the epoch of reionisation. Detailed studies with SPICA will enable a characterization of the dust and molecular content of these objects, constraining the scenarios of the formation of the first massive objects.

\section{CONCLUSION}

The combination of the next generation radio telescopes and observations with SPICA provide an excellent way to advance our understanding of the interplay between high and low energy phenomena that lead to the formation and evolution of AGN, galaxies and clusters of galaxies.

\section{ACKNOWLEDGEMENTS}

I am grateful for the collaborators that I have pleasantly worked with. Joe Lazio and Richard Schillizzi shared with me part of their SKA presentations. The core member of the LOFAR survey team, Peter Barthel, Philip Best, Marcus Brüggen, Gianfranco Brunetti, Krzysztof Chyzy, John Conway, Matt Jarvis, Matt Lehnert, George Miley, Raffaella Morganti, and Ignas Snellen were crucial in shaping the LOFAR survey science case. Finally, Cyril Tasse and Reinout van Weeren have given important input on Sections 6.2 and 6.3 .

\section{REFERENCES}

Bahcall, N. A., \& Fan, X. 1998, ApJ, 504, 1

Best, P., Longair, M. S., \& Röttgering, H. J. A. 1996, MNRAS, 280, L9

Croton, D. J., et al. 2006, MNRAS, 365, 11

De Breuck, C., van Breugel, W., Stanford, S. A., Röttgering, H., Miley, G., \& Stern, D. 2002, AJ, 123, 637

Fabian, A. C. 2009, ArXiv e-prints/0908.2784

Ferrari, C., Govoni, F., Schindler, S., Bykov, A. M., \& Rephaeli, Y. 2008, Space Science Reviews, 134, 93

Giovannini, G., \& Feretti, L. 2004, Journal of Korean Astronomical Society, 37, 323

Lazio, J. 2009, ArXiv e-prints/0910.0632

Ma, C., Ebeling, H., \& Barrett, E. 2009, ApJ, 693, L56

McCarthy, P. J. 1993, ARA\&A, 31, 639

Miley, G., \& De Breuck, C. 2008, A\&A Rev., 1

Röttgering, H. J. A., van Ojik, R., Miley, G., Chambers, K., van Breugel, W., \& de Koff, S. 1997, A\&A, 326, 505

Schilizzi, R. T., Dewdney, P. E. F., \& Lazio, T. J. W. 2008, in Society of Photo-Optical Instrumentation Engineers (SPIE) Conference Series, Vol. 7012, Society of PhotoOptical Instrumentation Engineers (SPIE) Conference Series

Seymour, N., et al. 2007, ApJS, 171, 353

Tasse, C., Best, P. N., Röttgering, H., \& Le Borgne, D. 2008, A\&A, 490, 893

van Breugel, W., de Breuck, C., Stanford, S. A., Stern, D., Röttgering, H. J. A., \& Miley, G. K. 1999, ApJL, 518, 61

van Weeren, R. J., Röttgering, H. J. A., Brüggen, M., \& Cohen, A. 2009, A\&A, 505, 991 